\title{
APPROXIMATION AND WEAK-STAR APPROXIMATION IN BANACH SPACES ${ }^{1}$
}

\author{
BY DAVID W. DEAN \\ Communicated by Robert Bartle, January 16, 1973
}

\begin{abstract}
If $X^{*}$ has a weak-star basis and if $X$ is separable, then $X$ has a basis. If $X^{*}$ has the weak-star $\lambda$-m.a.p. [a weak-star $\pi_{\lambda}$-decomposition], then $X$ has the $\lambda$-m.a.p. [a $\pi_{\lambda+\lambda^{2}+\varepsilon}$-decomposition]. If $X^{*}$ has a weak-star $\pi_{\lambda}$-decomposition and if $X$ is separable, then $X$ has a finite dimensional decomposition.
\end{abstract}

The problem of whether $X$ is separable if $X^{*}$ has a weak-star basis $[8$, p. 151] is unsolved, though there are candidates for a counterexample $[3,3.1]$, [6, pp. 243, 244]. In this note techniques developed in [5] are used, together with certain properties of weak convergence, to show that weak-star approximation methods in $X^{*}$ will yield approximation properties in $X$.

In [5] the authors established very deep relationships between approximation methods in a Banach space $X$ and its dual $X^{*}$. In particular they proved that if $X^{*}$ has a basis, then $X$ has a shrinking basis; and if $X^{*}$ is a $\pi_{\lambda}$-space, then $X$ is a $\pi_{\delta}$-space for some $\delta>1$. A fundamental tool in this work was the "principle of local reflexivity" [5], [6]. The basic corollary needed below is the following Theorem A $[5,3.1]$ or $[4$, p. 482], where $\mathscr{L}(B)$ is the space of bounded linear operators from $B$ to $B$.

THEOREM A. Let $T$ be a finite rank operator in $\mathscr{L}\left(X^{*}\right)$ and let $F \subset X^{*}$ have $\operatorname{dim} F<\infty$. Let $\varepsilon>0$. Then there is an $S$ in $\mathscr{L}(X)$ such that $S^{*}\left(X^{*}\right)$ $=T\left(X^{*}\right), f(S x)=T f(x)$ for each $f$ in $F, x$ in $X$, and $\|S\| \leqq(1+\varepsilon)\|T\|$. If $T$ is a projection, then taking $F$ to include $T\left(X^{*}\right), S$ is a projection.

THEOREM 1. Let $\left(T_{\alpha}\right)$ be a net of finite rank operators in $\mathscr{L}\left(X^{*}\right)$ such that $\left\|T_{\alpha}\right\| \leqq \lambda$ for all $\alpha$ and $\lim T_{\alpha} f(x)=f(x)$ for each $f$ in $X^{*}, x$ in $X$. Then there is a net of finite rank operators $\left(S_{\beta}\right)$ in $\mathscr{L}(X)$ such that $\lim S_{\beta} x=x$ for each $x,\left\|S_{\beta}\right\| \leqq \lambda$ for each $\beta$.

PrOOF. For each finite-dimensional subspace $F$ of $X^{*}$, use Theorem A to find $S_{\alpha, F}$ such that $f\left(S_{\alpha, F} x\right)=T_{\alpha} f(x)$ for every $f$ in $F, x$ in $X, S_{\alpha, F}^{*}\left(X^{*}\right)$ $=T_{\alpha}\left(X^{*}\right)$ and $\left\|S_{\alpha, F}\right\| \leqq \lambda(1+1 /(1+\operatorname{dim} F))$. Let $\left(\alpha_{1}, F_{1}\right) \geqq\left(\alpha_{2}, F_{2}\right)$, if $\alpha_{1} \geqq \alpha_{2}, F_{1} \supset F_{2}$. Then $(1+\operatorname{dim}(F)) S_{\alpha, F} /(2+\operatorname{dim}(F))=R_{\alpha, F}$ has norm $\leqq \lambda$ and $\lim f\left(R_{\alpha, F} x\right)=f(x)$ for every $f$ in $X^{*}, x$ in $X$. Then a $\operatorname{net}\left(P_{\beta}\right)$ of convex combinations of $\left(R_{\alpha, F}\right)$ has the property that $\lim P_{\beta} x=x$ for

AMS (MOS) subject classifications (1970). Primary 46B15, 46A20; Secondary 47A65.

${ }^{1}$ This work was partially supported by the Battelle Advanced Studies Center, Geneva. 
each $x$ (using [2, p. 477], for example).

REMARK 1 . If $X$ is separable and if $\left(x_{n}\right)$ is dense in $X$, then choosing $\alpha_{1}$ such that $\left\|P_{\alpha_{1}} x_{1}-x_{1}\right\|<1$ and $\alpha_{n+1}>\alpha_{n}$ such that $\left\|P_{\alpha_{n+1}} x_{i}-x_{i}\right\|$ $<1 /(n+1)$ when $i \leqq n+1$, one constructs a sequence $S_{n}=P_{\alpha_{n}}$ such that $S_{n} x \rightarrow x$ for each $x$.

THEOREM 2. Let $\left(T_{\alpha}\right)$ be a net of finite rank projections in $\mathscr{L}\left(X^{*}\right)$ such that $T_{\alpha}\left(X^{*}\right) \supset T_{\beta}\left(X^{*}\right)$, if $\alpha>\beta$ and $\lim T_{\alpha} f(x)=f(x)$ for each $f$ in $X^{*}$, $x$ in $X$. Let $X$ be separable. Then $X$ has a finite-dimensional decomposition.

Proof. If $\left(T_{\alpha}\right)$ is a sequence $\left(T_{n}\right)$, the proof proceeds easily from $[5,4.1]$ as follows. Set $Y=\bigcup T_{n}\left(X^{*}\right)$. Then $T_{n} y \rightarrow y$ for each $y$ in $Y$ and, choosing $S_{n}$ as in Remark 1, the conditions of 4.1(c) in [5] are satisfied. For the general case choose $\left(S_{n}\right)\left(=P_{\alpha_{n}}\right)$, as in Remark 1 above, such that $\left(S_{n}\right)$ has the following property: If

$$
P_{\alpha_{n}}=\sum_{1}^{k_{n}} a_{i} R_{\left(\alpha_{i}, F_{i}\right)} \quad \text { and } \quad P_{\alpha_{n+1}}=\sum_{1}^{k_{n+1}} b_{i} R_{\left(\beta_{i}, G_{i}\right)}
$$

then

$$
\left(\alpha_{i}, F_{i}\right) \leqq\left(\beta_{j}, G_{j}\right) \text { for each } i, j, n
$$

(e.g. $\left[2\right.$, p. 477] or $\left[1\right.$, p. 40]). Further, choose the $R_{\alpha, F}$ to be projections such that $R_{\alpha, F}^{*}\left(X^{*}\right)=T_{\alpha}\left(X^{*}\right)$, as promised in Theorem A above. Let $R_{n}=R_{\left(\alpha_{i}, F_{i}\right)}$, where $\left(\alpha_{i}, F_{i}\right)$ is larger than the indices in $\sum_{1}^{k_{n}} a_{j} R_{\left(\alpha_{j}, F_{j}\right)}$. Then $Q_{n}=R_{n}+P_{\alpha_{n}}-R_{n} P_{\alpha_{n}}$ is a projection such that $Q_{n}^{*}\left(X^{*}\right)=R_{n}^{*}\left(X^{*}\right)$ $=R_{\left(\alpha_{i}, F_{i}\right)}^{*}\left(X^{*}\right)=T_{\alpha_{i}}\left(X^{*}\right)$, and $Q_{n+1}^{*}\left(X^{*}\right) \supset Q_{n}^{*}\left(X^{*}\right)$ for each $n$. This is computed in the proof of the Theorem 3 below using the method in [5, Lemma 4.3]. Set $Y=\bigcup Q_{n}^{*}\left(X^{*}\right)$ and apply $[5,4.1(\mathrm{c})]$.

Corollary 1. Let $X^{*}=\sum_{1}^{\infty} Y_{i}$, where each $Y_{i}$ is finite dimensional, and for $x^{*}$ in $X^{*}$ there is a unique sequence $\left(f_{i}\right), f_{i} \in Y_{i}$, such that $\lim _{n} \sum_{1}^{n} f_{i}(x)$ $=x^{*}(x)$ for every $x$ in $X$. If $X$ is separable, then $X$ has a finite-dimensional decomposition.

Proof. The partial sum projections $V_{n}\left(X^{*}\right)=\sum_{1}^{n} Y_{i}$ are uniformly bounded [8, pp. 147-149]. Set $Y=\bigcup V_{n}\left(X^{*}\right)$. Then $Y$ is separable and, by Theorem 2, $X$ has a finite-dimensional decomposition.

Corollary 2. Let $X^{*}$ have $w^{*}$-basis $\left(f_{n}\right)$ and suppose $X$ is separable. Then $X$ has a basis.

Proof. By hypothesis each $f$ in $X^{*}$ has an expansion $\sum_{1}^{\infty} a_{n} f_{n}$ where the convergence is in the $w^{*}$-topology. Set $V_{n} f=\sum_{1}^{n} a_{n} f_{n}$. Let $R_{n, F}$ be a projection on $X$ as in Theorem A, such that $R_{n, F}^{*}\left(X^{*}\right)=V_{n}\left(X^{*}\right)=\left[f_{1}, \ldots\right.$, 
$\left.f_{n}\right]$. Then $\lim _{(n, F)} f\left(R_{n, F} x\right)=f(x)$ for every $f$ in $X^{*}$. Since $X$ is separable, a sequence of convex combinations $\left(P_{n}\right)$ of $\left(R_{n, F}\right)$ converges strongly $\left(\lim P_{n} x=x\right)$ to the identity, and $P_{j}^{*}\left(X^{*}\right) \subset\left[f_{n}\right]$. Since $V_{n} y \rightarrow y$ for all $y$ in $\left[f_{n}\right]$ and $P_{n} x \rightarrow x$ for all $x$ in $X$, Theorem 4.1 in [5] yields that $X$ has a finite-dimensional decomposition given, say, by $\left(Q_{n}\right)$. Moreover, $Q_{n}^{*}\left(X^{*}\right)$ is $\varepsilon$-close to some $V_{k(n)}\left(X^{*}\right)[5,4.9]$. This assures that $\left\{\left(Q_{n+1}^{*}-Q_{n}^{*}\right)\left(X^{*}\right)\right\}$ have bases with uniformly bounded basis constants [5, p. 501], and so $\left\{\left(Q_{n+1}-Q_{n}\right) X\right\}$ have bases with uniformly bounded basis constants [5, p. 502]. Thus, $X$ has a basis (e.g., [5, Lemma 2.2]).

W. B. Johnson, in conversation with the author, observed that the methods above, together with the proof of Lemma 4.3 in [5], yield the following theorem.

THEOREM 3. Let $\left(T_{\alpha}\right)$ be a net of finite rank projections such that $\left\|T_{\alpha}\right\| \leqq \lambda$ for every $\alpha$ and such that if $\alpha>\beta$, then $T_{\alpha} X^{*} \supset T_{\beta} X^{*}$. Suppose further that $\lim _{\alpha} T_{\alpha} f(x)=f(x)$ for each $f$ in $X^{*}$ and $x$ in $X$. Then $X$ is a $\pi_{\lambda^{2}+2 \lambda+\delta}$ space for each $\delta>0$.

PRoOF. If the $\left(T_{\alpha}\right)$ of Theorem 1 are projections with $T_{\alpha}\left(X^{*}\right) \supset T_{\beta}\left(X^{*}\right)$ when $\alpha>\beta$, and if the $R_{\alpha, F}$ in the proof of Theorem 1 are chosen to be projections such that $R_{\alpha, F}^{*}\left(X^{*}\right)=T_{\alpha}\left(X^{*}\right)$, let $\left(U_{\beta}\right)$ be the corresponding net of finite rank operators such that $\lim U_{\beta} x=x$ for every $x$. If $U_{\beta}$ $=\sum_{1}^{n} a_{i} R_{\alpha_{i}, F_{i}}$, where $\left(\alpha_{1}, F_{1}\right)<\cdots<\left(\alpha_{n}, F_{n}\right)$, let $S_{\beta}=R_{\alpha_{n}, F_{n}}+U_{\beta}-$ $R_{\alpha_{n}, F_{n}} U_{\beta}$. Then

$$
S_{\beta}^{*}\left(X^{*}\right)=R_{\alpha_{n}, F_{n}}^{*}\left(X^{*}\right)+U_{\beta}^{*}\left(X^{*}\right)-U_{\beta}^{*} R_{\alpha_{n}, F_{n}}^{*}\left(X^{*}\right) \subset T_{\alpha_{n}}\left(X^{*}\right)
$$

and

$$
S_{\beta}^{*} T_{\alpha_{n}} x^{*}=T_{\alpha_{n}} x^{*}
$$

for each $x^{*}$ in $X^{*}$. Thus, $S_{\beta}^{*}$ is a projection onto $T_{\alpha_{n}}\left(X^{*}\right)$. It follows that $\left(S_{\beta}\right)$ is a net of projections. Moreover, if $\left\|U_{\beta} x-x\right\|<\delta$, then

$$
\begin{aligned}
\left\|S_{\beta} x-x\right\| & =\left\|R_{\alpha_{n}, F_{n}} x+U_{\beta} x-R_{\alpha_{n}, F_{n}} U_{\beta} x-x\right\| \\
& \leqq\left\|R_{\alpha_{n}, F_{n}}\right\|\left\|x-U_{\beta} x\right\|+\left\|U_{\beta} x-x\right\| \leqq(\lambda+\varepsilon+1) \delta
\end{aligned}
$$

so that $\lim S_{\beta} x=x$.

If $X$ has a finite-dimensional decomposition [basis], then $X^{*}$ has a weak-star finite-dimensional decomposition [weak-star basis]. If $X$ has a $\pi_{\lambda}$-decomposition [ $\lambda$-m.a.p.], then $X^{*}$ has the weak-star $\lambda$-m.a.p. It is not known to the author if $X^{*}$ has a weak-star $\pi_{\lambda}$-decomposition. An answer to this question will answer, via Theorem 2 , whether $X$ has a finitedimensional decomposition if $X$ is a separable $\pi_{\lambda}$-space.

\section{REFERENCES}

1. M. M. Day, Normed linear spaces, Springer-Verlag, Berlin; Academic Press, New York, 
1962. MR 26 \#2847.

2. N. Dunford and J. T. Schwartz, Linear operators. I: General theory, Pure and Appl. Math., vol. 7, Interscience, New York, 1958. MR 22 \# 8302.

3. J. A. Dyer, The mean Stieltjes integral representation of a bounded linear transformation, J. Math. Anal. Appl. 8 (1964), 452-460. MR 28 \# 4079.

4. William B. Johnson, On the existence of strongly series summable Markuschvich bases in Banach spaces, Trans. Amer. Math. Soc. 157 (1971), 481-486. MR 43 \# 7914.

5. W. B. Johnson, H. P. Rosenthal and M. Zippin, On bases, finite dimensional decompositions and weaker structures in Banach spaces, Israel J. Math. 9 (1971), 488-506. MR 43 \#6702.

6. J. Lindenstrauss and H. P. Rosenthal, The $\mathscr{L}_{p}$-spaces, Israel J. Math. 7 (1969), 325-349. MR 42 \# 5012.

7. H. P. Rosenthal, On injective Banach spaces and the spaces $L^{\infty}(\mu)$ for finite measures $\mu$, Acta Math. 124 (1970), 205-248. MR 41 \# 2370.

8. I. Singer, Bases in Banach spaces. Vol. 1, Die Grundlehren der math. Wissenschaften, Band 154, Springer-Verlag, Berlin and New York, 1970.

Department of Mathematics, Ohio State University, Columbus, Ohio 43210 